\title{
Full-field pulsed magnetophotoelasticity - a description of the instrument
}

\author{
S Gibson $^{1 *}$, G W Jewell ${ }^{2}$, and R A Tomlinson ${ }^{1}$ \\ ${ }^{1}$ Department of Mechanical Engineering, University of Sheffield, Sheffield, UK \\ ${ }^{2}$ Department of Electronic and Electrical Engineering, University of Sheffield, Sheffield, UK
}

The manuscript was received on 17 November 2004 and was accepted after revision for publication on 26 October 2005.

DOI: $10.1243 / 03093247 J S A 92$

\begin{abstract}
This paper describes a novel instrument used for the analysis of full-field throughthickness stress distributions using the theory of magnetophotoelasticity (MPE) developed by Aben [1] and developed by Clarke et al. [2, 3]. MPE is an experimental stress analysis technique that involves the application of a magnetic field parallel to an electromagnetic wave propagating through a birefringent model within a polariscope. The effect viewed through the polariscope is then a combination of the model's birefringence and the Faraday rotation created in the model by the magnetic field. Aben developed this technique especially for use in the measurement of stress profiles where the integrated photoelastic pattern alone yields little information. Clarke et al. developed MPE in order to study toughened glass.

To date, the technique of MPE has been a single-point measurement, which is of limited utility in the investigation of three-dimensional stress in toughened glasses. The pulsed magnetopolariscope (PMP), described here, enables the full-field application of MPE. This paper contains a description of the novel apparatus and demonstrations used to validate the performance of a proof-of-concept PMP instrument. The paper also highlights improvements in the application of MPE, which are now possible with this new equipment. These improvements include the extension of MPE to larger areas of analysis, three-dimensional stress analysis, and the possibility of analysing a general unknown stress distribution.
\end{abstract}

Keywords: three-dimensional photoelasticity, residual stress measurement, toughened glass

\section{INTRODUCTION}

It is generally accepted that the stress profile through the thickness of a thermally toughened glass plate is approximately parabolic, with tensile stress in the mid-plane and compressive stress at the surfaces. Errors within the manufacturing process may cause this profile to become asymmetric, resulting in tensile stresses closer to the surface, thus weakening the glass. If a scratch were made in the glass and this penetrated into the tensile region, the residual stress would act to open the flaw into a larger and potentially catastrophic crack. The glass industry wishes to be able to identify these errors, so preventing a potential product recall and enabling corrective action to be taken before the glass leaves

\footnotetext{
* Corresponding author: Department of Mechanical Engineering, University of Sheffield, Mappin Building, Mappin Street, Sheffield, S1 3JD, UK. email: sarah.gibson@sheffield.ac.uk
}

the factory. Currently there are few techniques that have the potential to make this measurement and those regularly used by manufacturers cannot detect the full through-thickness profile in which they are interested. Traditional integrated photoelastic analysis is of limited utility in analysing such toughened glass sheets, due to this three-dimensional stress field. Sectioning glass in order to conduct twodimensional photoelastic analysis is hazardous and would result in stress relief around any cut, which would mask the real stress profile. The throughthickness stress distribution can, however, be found using a technique called magnetophotoelasticity (MPE).

MPE was proposed by Aben [1] and developed by Clarke et al. $[2,3]$ to enable some measurements of through-thickness residual stress distributions. In MPE a magnetic field is applied across a birefringent component within a polariscope. The magnetic field causes rotation of the light vector as it travels through 


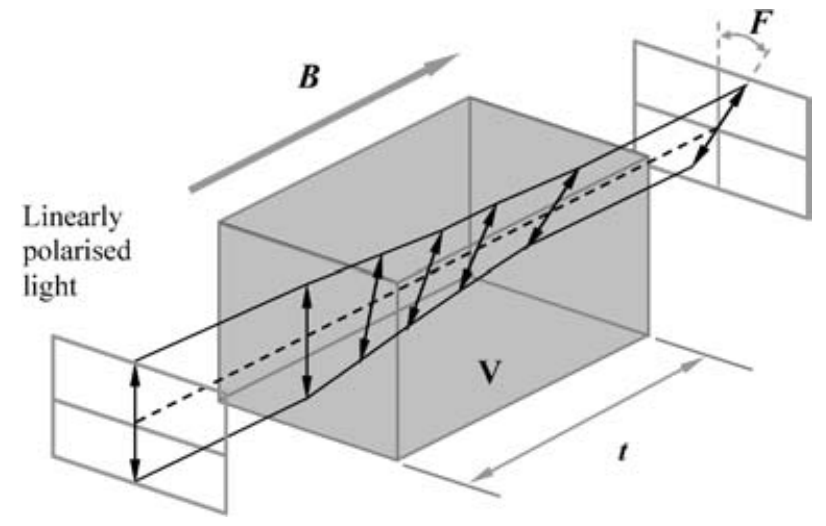

Fig. 1 Schematic diagram describing the Faraday rotation in a stress-free medium

the sample parallel to the magnetic field. This phenomenon is known as the Faraday effect. Where the light is polarized the polarity rotates as the electromagnetic wave propagates through the medium (see Fig. 1). The rotation in polarity, $F$, caused by the Faraday effect, depends on the Verdet constant $V$ (which is a function of the material and the wavelength of the light), the magnetic flux density $B$, and the thickness of the medium $t$. This relationship is shown by

$$
F=\mathrm{V} B t
$$

If a stressed medium is examined within a magnetic field and in a polariscope the final effect viewed through the apparatus is a combination of birefringence and the Faraday rotation within the sample. From this combined effect more information about the stress distribution within the model can be determined. The characteristic photoelastic parameters (the characteristic retardation and the two characteristic directions [4]) are determined through the point of interest with and without the magnetic field (Aben's method uses only the collection of character- istic parameters with the magnetic field). These values can then be used in an algorithm, which assumes an a priori stress profile to determine the stress distribution present through the sample thickness [3]. Clarke et al. developed MPE for measurements in glass and the technique was used at Pilkington plc for a number of years. However, due to several experimental difficulties the technique has not been used recently [3].

This paper highlights some limitations of MPE due to the single-point apparatus used previously for its application and describes the full-field pulsed magnetopolariscope (PMP), which overcomes some of these limitations. The PMP development will make the technique of MPE more acceptable for use in an industrial environment. The paper concludes with a description of a simple demonstration using the instrument. A companion paper describes experiments performed with a proof-of-concept instrument [5].

\section{THE LIMITATIONS OF A SINGLE-POINT MAGNETOPOLARISCOPE}

The existing apparatus, available to measure the through-thickness distribution of in-plane stresses, is called the magnetopolariscope and a schematic of this is shown in Fig. 2. The magnetopolariscope contains a water-cooled, half-yoke, iron-cored electromagnet. A $4 \mathrm{~mm}$ diameter hole is bored through the electromagnet poles to create a passage for light from a laser. Test samples can be positioned between the magnet poles. A polarizer, analyser, and a removable quarter-wave plate are adjusted using stepper motors in order to determine the photoelastic characteristic parameters. This apparatus was used to prove that MPE is useful as a diagnostic tool in

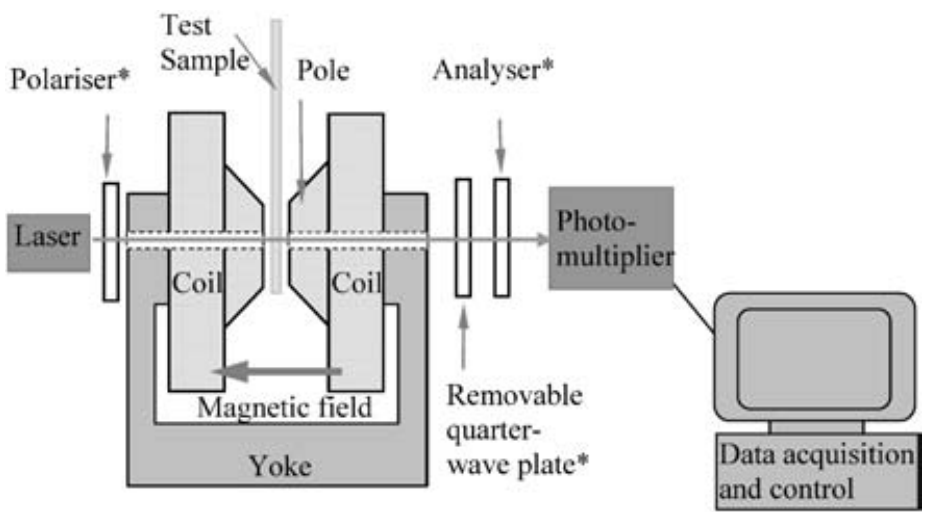

Fig. 2 Schematic diagram of the single-point magnetopolariscope (*Positioned using stepper motors) 
toughened automotive glass $[\mathbf{3}, \mathbf{6}]$. However, this apparatus has several limitations, which are discussed below.

1. Degree of Faraday rotation. Aben, and later Clarke et al. suggested that increasing the magnetic flux density created by the magnetopolariscope would improve the accuracy of MPE $[\mathbf{1}, \mathbf{3}]$. It is simple to demonstrate that an increase in the magnitude of the Faraday rotation through the application of a higher magnetic field will decrease the sensitivity of MPE to experimental errors [7]. However, the maximum achievable magnetic flux density in the air gap of an ironcored laboratory electromagnet is usually limited by saturation of the yoke to $\sim 2.5 \mathrm{~T}$, with any further increase being gained with severely diminished returns in terms of increasing current. This field strength gives rise to a relatively small Faraday rotation.

2. Area of data collection. A single-point measurement method may fail to detect an area of problematic stress and so might not provide an adequate check on the strength of the glass. The ability to collect comparable data in a single fullfield reading would overcome this limitation.

3. Speed of analysis. If the user is proficient, the measurement of the principal stress difference in a glass sample using the single-point magnetopolariscope takes about 65 minutes. Of this time, the experimental measurements take approximately 50 minutes while processing the characteristic parameters to find the principal stress difference through the thickness of the sample can take up to 15 minutes. The speed of these measurements is obviously a disincentive to the adoption of the technique in industry.

4. Assumed stress profile. Although the throughthickness stress distribution in toughened glass is generally accepted to be approximately parabolic, this may not be true at the glass edges where edgecooling effects begin to influence the distribution
[8]. Glass manufacturers are also interested in the measurement of other types of stress distribution, such as those observed in laminated and chemically toughened glasses where the stress distributions are less well understood. The algorithm used in references [2] and [3] contains an assumption that the shape of the stress distribution is known. This assumption limits the investigation of more general stress distributions. The ability to measure a general stress profile would make MPE more useful for research and development of new glass products as well as for product control.

\section{THE APPARATUS OF THE PULSED MAGNETOPOLARISCOPE}

It was recognized that only small performance increments could be achieved by optimizing the existing electromagnet system, with no prospect of realizing a practically useful tool for large components. In order to overcome the limitations of the single-point magnetopolariscope discussed above, a full-field magnetopolariscope capable of nondestructive, rapid determination of internal stresses in engineering components has been developed. The novel apparatus was named the pulsed magnetopolariscope (PMP) and a schematic of this idea is shown in Fig. 3 [9].

This apparatus (Fig. 3) uses a pair of split 'aircored' solenoid coils, a polariscope, a capacitordischarge magnetizer, and a high-speed video camera. Light from a stable light source is directed through the first polarizer and solenoid coil, through the sample, and then it proceeds through the second solenoid coil and polarizer to the high-speed video camera. A removable quarter-wave plate can also be placed between the first polarizer and the sample. The magnetizer consists of a large bank of capacitors which are charged over a period of several seconds and then discharged through the solenoids to produce

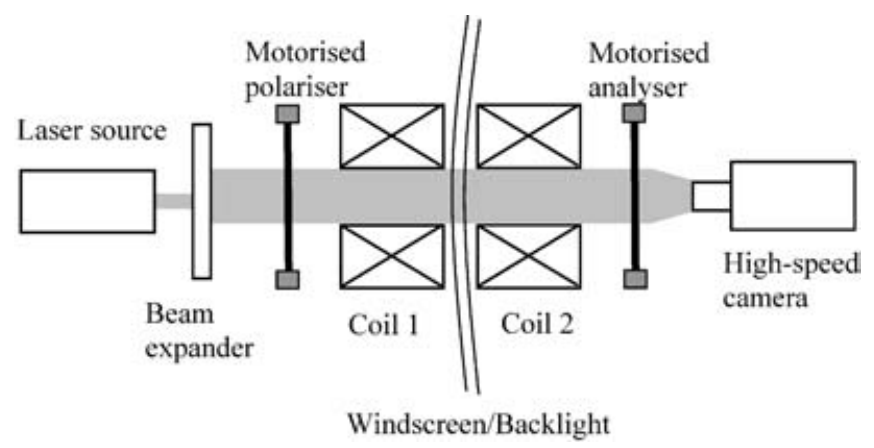

Fig. 3 Schematic diagram of the proposed design of the full-field pulsed magnetopolariscope 
a high-intensity pulsed magnetic field in the area between the split coils, with a typical pulse duration of a few milliseconds. The camera is triggered to record the intensity variation over the discharge period. This type of system has several advantages over the single-point equipment, including an increased magnetic flux density, an increased field of view, and a near-homogeneous magnetic field over the area of measurement. The equipment also produces constant magnetic flux density through the thickness of the sample. The split coil allows large samples to be analysed non-destructively.

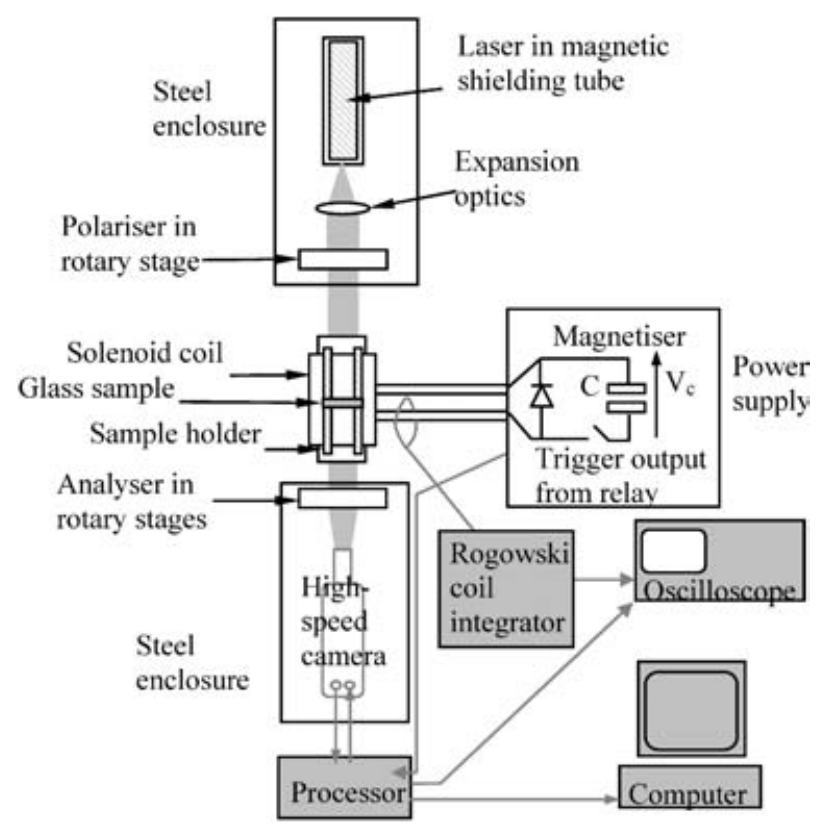

Fig. 4 Schematic diagram of the proof-of-concept pulsed magnetopolariscope
A proof-of-concept pulsed magnetopolariscope (PMP) was assembled, using a single solenoid coil, which was readily available for the purposes of proving the concept of the instrument. The single coil proof-of-concept PMP instrument is shown schematically in Fig. 4 and photographically in Fig. 5, and is described more fully below.

\subsection{Magnetizer unit and solenoid}

The capacitor-discharge magnetizer (M20kJA made by Hirst Magnetic Instruments Limited) can store up to $20 \mathrm{~kJ}$ of energy, has a maximum output voltage of $3000 \mathrm{~V}$, and has a maximum current capability of $30000 \mathrm{~A}$. An existing coil previously employed in the magnetization of permanent magnets was used [10]. The solenoid coil has a resistance of $48 \mathrm{~m} \Omega$ and an inductance of $1.07 \mathrm{mH}$, a bore of $70 \mathrm{~mm}$ diameter and an axial length of $144 \mathrm{~mm}$, with 148 turns of copper wire of square cross-section $5 \mathrm{~mm} \times 5 \mathrm{~mm}$. The copper wire is wound around a non-magnetic, non-conducting tube and successive layers are wound in the same sense. The copper wire is then over-wrapped with a filament epoxy composite to counteract the large mechanical forces on the coil conductors. The magnetic flux density measured in the bore of the solenoid coil is shown on the first axis of Fig. 6. The magnetic flux rapidly increases to its maximum value over about $2.5 \mathrm{~ms}$ and then reduces more gradually. The system produces a maximum flux density of $4.11 \mathrm{~T}$ in the central bore of the solenoid - almost double that which can be achieved using the iron-yoke electromagnet in the single-point magnetopolariscope. Appropriately rated capacitordischarge systems are capable of producing much

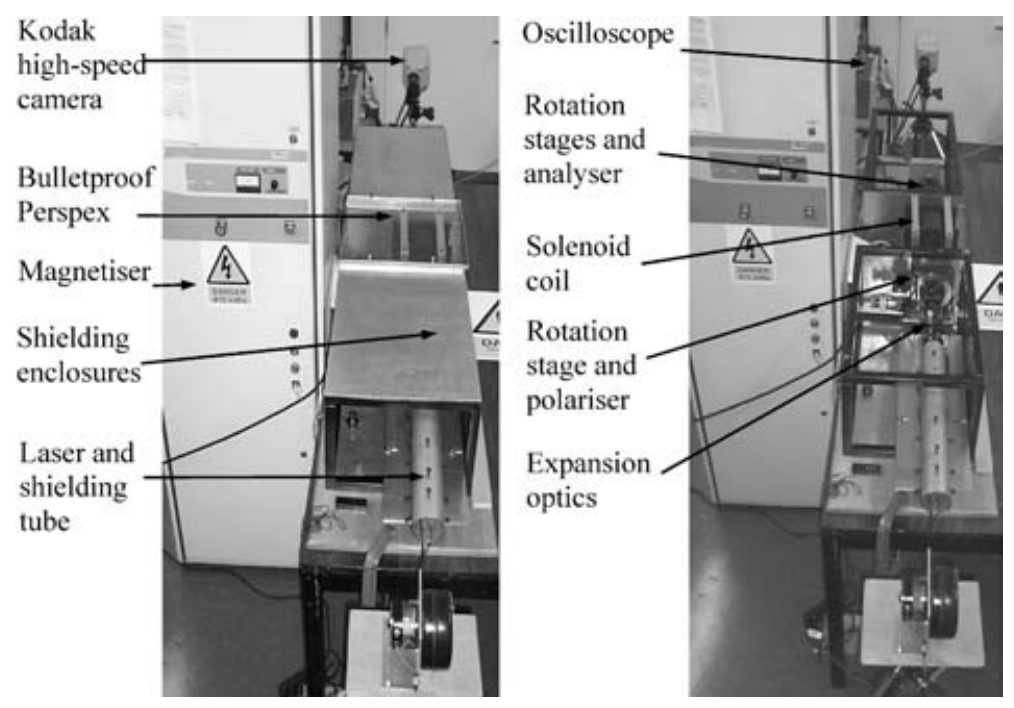

Fig. 5 Showing the proof-of-concept pulsed magnetopolariscope with and without the shielding enclosures. These are used to protect the components from the high magnetic fields 


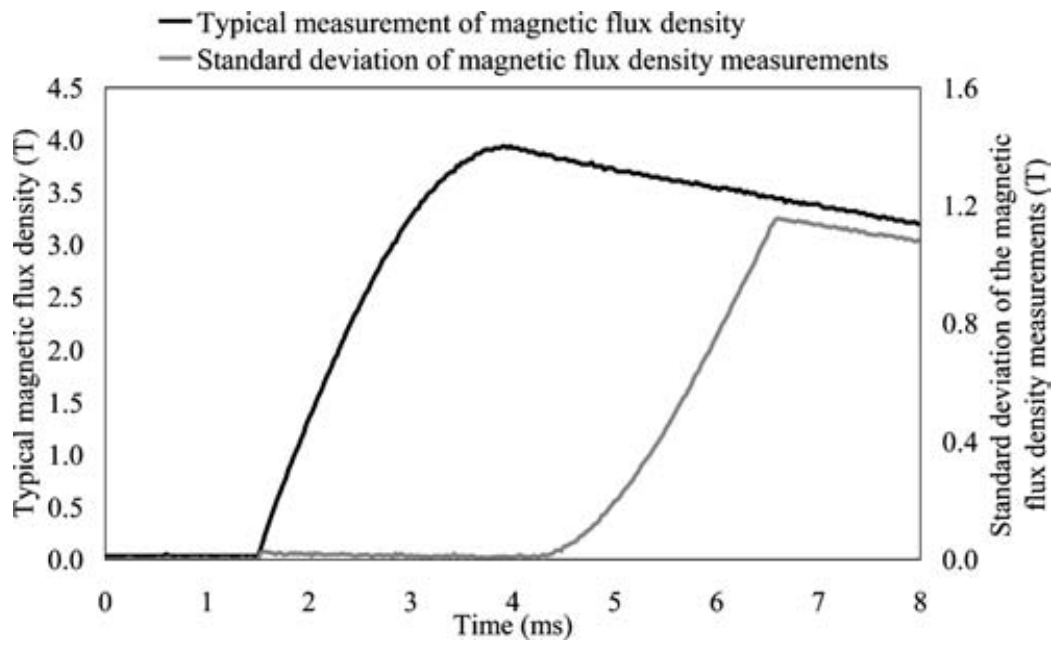

Fig. 6 Showing the magnetic flux density in the solenoid coil during one pulse of the solenoid and on the second axis the standard deviation between 10 of these measurements

higher fields, with $10 \mathrm{~T}$ being readily achievable with industrial equipment [11] and up to $50 \mathrm{~T}$ for highly specialized scientific instruments [12].

\subsection{Image capture and flux density measurement}

A Kodak HS 4540 high-speed video system was used to capture the variation in intensity of the light from the PMP throughout the magnetic pulse, at a frame rate of 40500 (frames per second) and a resolution of $64 \times 64$ pixels. The frames were collected and then downloaded from the camera on to a personal computer; the download time was comparatively slow at approximately 6 minutes for 102 frames. The download rates and resolution of more modern cameras are considerably higher and would greatly assist the viability of the instrument for rapid testing.

The variation in magnetic flux density during the pulse was derived from a calibrated scaling of the coil current waveform, which in turn was measured using a Rogowski coil and a digital storage oscilloscope. In order to synchronize the captured images with the variation in the magnetic field, a trigger signal generated within the magnetizer was employed to reference precisely the current waveform captured by a digital storage oscilloscope and the sequence of camera images.

Since the proposed measurement technique is reliant on obtaining a sequence of measurements at different polarizer positions, it was important to establish the repeatability of the magnetic field pulse, particularly as this is in essence an 'open-loop' system in which the initial capacitor voltage is controlled rather than the current itself. Hence, an experiment was performed to gauge the repeatability of the magnetic pulse system. The current in the magnetizer was discharged through the solenoid coil 10 times and for each a voltage reading from the Rogowski coil integrator was recorded and the corresponding magnetic flux density calculated. The standard deviation of 10 corresponding magnetic flux readings is plotted in Fig. 6 along with a typical magnetic flux time curve. Figure 6 shows that the initial part of the pulse is very repeatable as the standard deviation is low, but after the peak in the magnetic pulse the standard deviation increases. As a result of this measurement it was determined that to achieve a high repeatability only measurements made during the initial rise time of the pulse should be used.

\subsection{Magnetic enclosures}

The stray magnetic field produced by the coil could have a detrimental effect on the electrical components used for the data collection, including the camera and the laser. Steel shielding enclosures were fabricated to screen the laser, expansion optics, and rotation stages from the stray field of the coil, while the other electrical elements were kept as far from the coil as was practically possible. Measurements of flux density were taken during the magnetic pulse with a Gaussmeter located within the enclosures; these are shown in Fig. 7. The magnetic flux measured in the light source enclosure (thin grey line in Fig. 7) and the camera enclosure (thin black line in Fig. 7) were low; however, without more testing it was difficult to establish an acceptable level. As a result of this uncertainty a steel tube was positioned around the laser and the resulting measurement, plotted in a thick grey line, reduced the magnetic flux density to below $0.03 \mathrm{~T}$ (i.e. a negligible amount). The high-speed camera was moved further away from the 


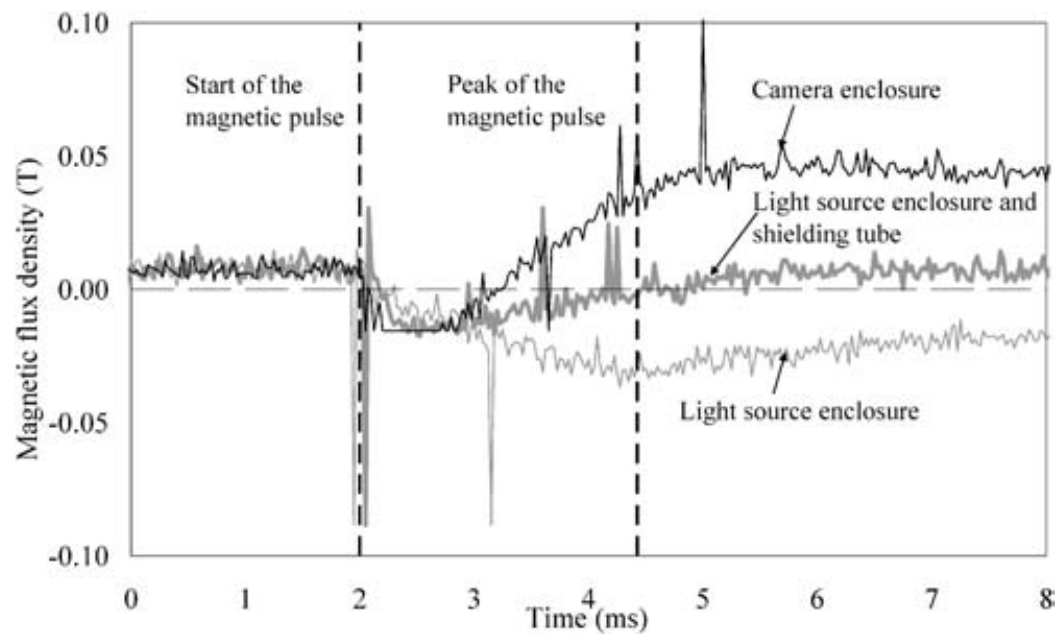

Fig. 7 Showing the magnetic flux density, created during the magnetic pulsing of the solenoid coil, at different locations in the apparatus. Each plot is marked to identify its location within the apparatus

solenoid coil to be certain of removing any magnetic effect on it.

\subsection{Description of the rotation stages}

As the characteristic photoelastic parameters in glass are small, a high degree of accuracy is required in their measurement and therefore the precision of the apparatus used is important. Two rotation stages were used to position the polarizer and analyser. These have a resolution of $0.001^{\circ}$, an origin repeatability of $0.001^{\circ}$, an absolute accuracy of $0.023^{\circ}$, and a maximum speed of $20 \mathrm{deg} / \mathrm{second}$. The rotation stages were controlled using an ESP-300 motion controller provided by Newport ${ }^{\odot}$.

\subsection{Description and testing of the light source}

In selecting a light source for this application, both the long- and short-term stability were important. Fourier polarimetry $[13,14]$ was used to collect automatically the full-field integrated photoelastic characteristic parameters used in full-field MPE and this technique is discussed in a companion paper [5]. Using Fourier polarimetry with the PMP requires the collection of 36 or 72 intensity images. At present this process takes up to two days because of the need to allow the coil to cool between successive pulses. This length of time could be considerably reduced in a future instrument where a cooling system could be used to enable the cycle time to be reduced to a few seconds, as is done frequently in industry. The images collected need to have the same source intensity so each of the images is only affected by the birefringence of the measured sample, the Faraday effect, and the position of the polarizers. If there is also some change in the intensity of the light source this will have a detrimental effect on the measurements taken. It would be an advantage if all the data required could be collected quickly or indeed in only two pulses of the magnet, as could be done using phase stepping suggested by Tomlinson and Patterson [15], but unfortunately this is not accurate enough for this application. For this reason experiments were performed to test a number of light sources and from these experiments a green laser of wavelength $543 \mathrm{~nm}$ was chosen as the most appropriate, since this laser produced the least intensity change over time and furthermore has the advantage of a shorter wavelength, which will increase the Faraday rotation (a property of optical activity [4]). An expander and a collimating lens were used to create light with good collimation.

A test was completed to investigate the longerterm stability of the laser and the effect of the magnetic field on its stability. In the first $7 \frac{1}{2}$ hours a frame was taken from a sequence of images every 15 minutes and immediately after a pulse of the magnet. The test was repeated without pulsing the magnet. An image created by the laser was recorded using the high-speed video camera at a frame rate of $40500 \mathrm{fps}$. The average intensity of the pixels over an area of $4 \times 4$ pixels (at a resolution of 4.3 pixels $/ \mathrm{mm}$ ) was taken from each frame and these values were normalized with the largest value detected. Figure 8 shows the results of this two-day stability test. The stability of the laser is not affected by the magnetic field. Once the laser is stabilized after about 50 minutes there is very little variation in its intensity over the $7 \frac{1}{2}$ hours. This stability is essential for the application of the methodology. 


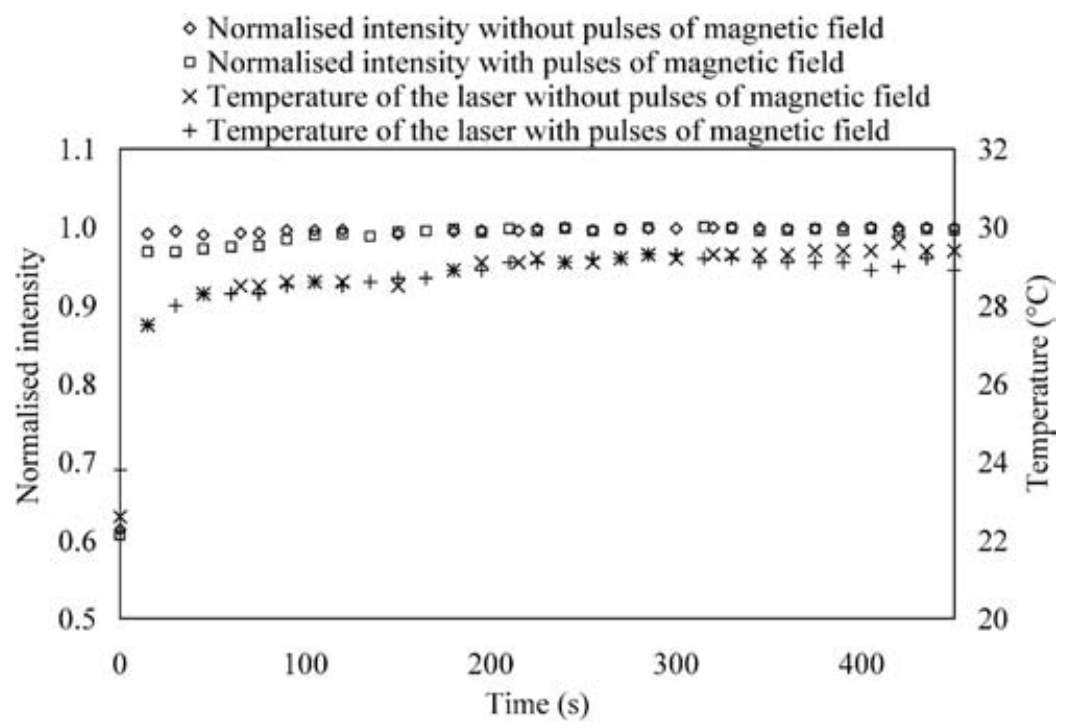

Fig. 8 Showing the change in normalized intensity of a laser over a period of $7 \frac{1}{2}$ hours and the temperature of the laser both with and without regular pulses of the magnetic field

Using the proof-of-concept PMP an experiment was conducted to test the intensity change during measurement of the characteristic parameters. Measurement of the full-field characteristic parameters using this instrument is made using a Fourier polarimetry method; this is explained more fully in a companion paper [5]. During Fourier polarimetry measurements, images are collected at different positions of the polarizers. Using the PMP a sequence of images is collected during the magnetic pulse at each of these polarizer positions. An image is extracted from each sequence at a desired magnetic flux density. These images are combined to find the characteristic parameters. In order to test the intensity change during real measurements, a toughened glass sample was positioned within the solenoid, and
18 sequences of frames each with the polarizer and analyser at different angles were collected. After 7 hours the experiment was repeated. The difference between these pairs of measurements was calculated. An average of these 18 differences is plotted in Fig. 9. The average difference between these readings has been found to be 5 out of $0-255$ grey levels of an 8 bit camera and the maximum difference was approximately 20 . This variation of about $2-8$ per cent is therefore relatively small.

The disadvantage of using a laser is that it produces speckle. It has been shown that a light source such as a mercury or sodium discharge lamp is more suitable for the application of Fourier polarimetry [9]. However, from the light sources available only a laser has sufficient constancy of intensity over the

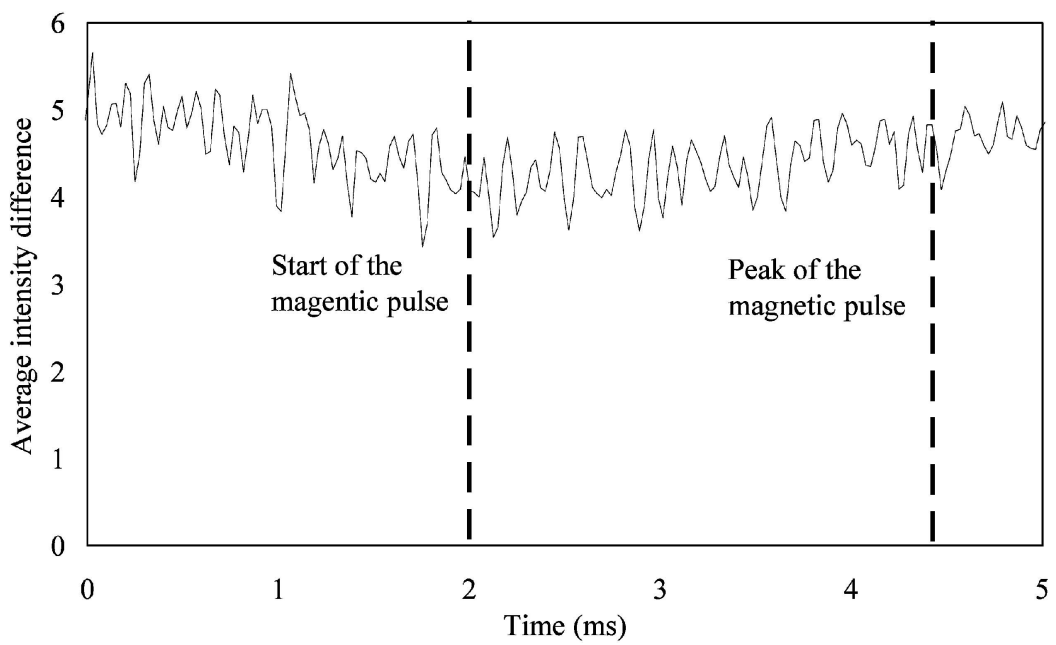

Fig. 9 Showing the average difference in intensity between 18 duplicate sequences of images taken at 18 different positions of the polarizers 
short time frame of the magnetic pulse and also over the longer term required with the current apparatus. The laser is easy to collimate and also has the advantage of providing an intense light source in comparison with the lamp sources, which is important when using short exposure times. Despite the speckle present in the images produced using the laser, the accuracy of the method seems reasonable for a first attempt. If speckle is found to present a more significant problem than currently indicated, it may be possible to use a light source that is less coherent, such as a light emitting diode (LED) or a d.c. powered lamp type light source, which would produce fewer speckles.

\section{INITIAL VALIDATION OF THE PMP}

In a simple investigation, the polarizer and analyser of the PMP were set at $70^{\circ}$ to each other to ensure a large enough initial intensity reading for an effective demonstration of the system. The light intensity produced by a green light laser passing through the polariscope was measured with and without a stressfree glass sample inside the solenoid coil. The intensity variation with time measured during a magnetic pulse is plotted in Fig. 10. This shows that with no glass there is no intensity change with the magnetic field, as there can be no Faraday rotation when a material is not present. If the glass sample is located within the magnetic field it can be seen that the intensity increases with the magnetic flux density, indicating the increasing Faraday rotation. The peak of the intensity is coincident with the peak in the magnetic flux density. The figure also confirms that the triggering system is accurate, as the start of the magnetic pulse is coincident with the increase in the light intensity due to the Faraday rotation. The collection of full-field MPE data is presented in the companion to this paper [5].

\section{FUTURE DEVELOPMENT}

In the proof-of-concept PMP the specimen had to be specifically made to be the correct size to fit within the existing solenoid coil. To be useful for industry the measurement should be non-destructive. A prototype would require the use of a split-coil design so that large glass samples can be examined without sectioning them (as shown in Fig. 3). This design will involve a balance between cost, maximum field strength, size of analysis, and field spatial homogeneity.

Aben [1] and Theocaris and Gdoutos [16] suggested that by collecting the characteristic parameters at many different magnetic fields a number of simultaneous equations are produced and a general three-dimensional stress distribution, about which no a priori information is known, could be found. The PMP can be used to collect many images during a pulse of the magnetic field (the number depends on the camera speed and the duration of the pulse) and provides an opportunity to measure the characteristic parameters at a number of different magnetic field strengths and Faraday rotations (by Fourier polarimetry for example). Through the development of the PMP this multimagnetophotoelasticity is now practically, as well as theoretically, possible and will be part of the next stage of this research.

This multimagnetophotoelastic approach could potentially be applied to glass and also to the

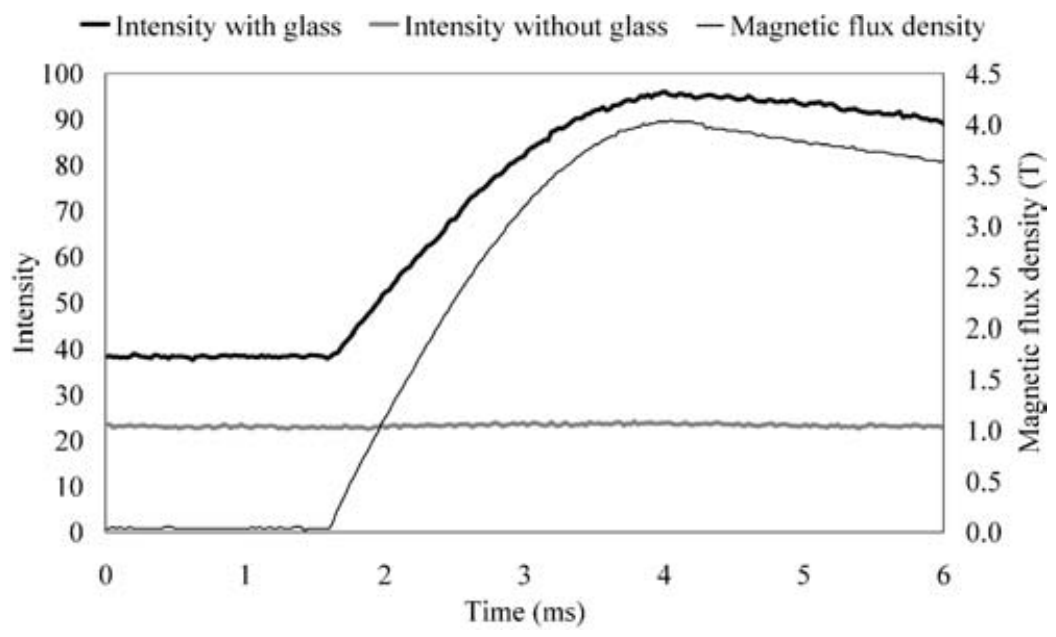

Fig. 10 Showing the measured intensity through the PMP with and without a stress-free sample of glass and both with an applied magnetic flux density 
measurement of three-dimensional stresses in epoxy models without the need to section them. This is an advantage over traditional three-dimensional photoelasticity where obtaining the full stress distribution often requires several models. These models are sectioned in different directions and both their examination and the stress reconstruction are time consuming.

\section{DISCUSSION}

The novel apparatus proposed here, named the pulsed magnetopolariscope, can be used to make full-field measurements of the through-thickness stress distribution in birefringent materials. The proof-of-concept instrument increases the magnetic flux density available to $4.11 \mathrm{~T}$, representing a 71 per cent increase on the $2.4 \mathrm{~T}$ created using the ironcored electromagnet. Given a magnetizer of sufficient stored energy, the field could be increased to $10-20 \mathrm{~T}$, but this would be costly. The system provides a homogeneous magnetic field across a region of diameter $70 \mathrm{~mm}$; this is over 300 times the area measured using the single-point apparatus. This larger area can be increased easily. The increased Faraday rotation obtained with the higher magnetic flux densities obtained using this equipment means that measurement errors relative to the magnitude of the characteristic parameters are reduced. The higher field strengths also make the technique easier to apply to thin glass.

Further work has been undertaken to develop a split coil design so that large samples can be examined. The new equipment could analyse a much larger area and would only be limited by the size of the capacitance available in the magnetizer and to a lesser extent the homogeneity of the magnetic field required.

There are several potential applications for the new equipment that are worth further investigation. The increase in the magnetic flux density means that the method of MPE could be applied to products such as annealed and laminated glass, which have lower stresses and would therefore benefit from higher magnetic fields and thus Faraday rotations. The equipment could also be applied to more general three-dimensional photoelasticity and general unknown stress profiles in glass by utilizing the information that can be collected at many magnetic flux densities during the magnetic pulse. The measurement of a general stress distribution would have advantages over scattered-light photoelasticity and the slicing of stress frozen models that are then examined by two-dimensional photoelasticity, which are the only methods currently able to measure three-dimensional stresses. The analysis of glass using both of these techniques is time consuming, and using two-dimensional photoelasticity is likely to be impossible.

\section{CONCLUSIONS}

In conclusion a proof-of-concept pulsed magnetopolariscope has been designed and assembled. The instrument was validated using the observation of the Faraday rotation in stress-free glass samples. Further experimental testing has been undertaken and is presented in a companion paper [5]. This new apparatus overcomes many of the limitations identified in the single-point magnetopolariscope and presents exciting possibilities for the future of fullfield three-dimensional photoelasticity.

\section{ACKNOWLEDGEMENTS}

The authors would like to thank Andrew Race and John Wilkinson for help with the triggering system, Richard Kay for building some of the apparatus, Pilkington plc and EPSRC for sponsorship of this project, and the EPSRC Engineering Instrument Loan Pool for the loan of the high-speed video camera.

\section{REFERENCES}

1 Aben, H. K. Magnetophotoelasticity - photoelasticity in a magnetic field. Expl Mechanics, 1970, 10, 97-105.

2 Clarke, G. P. Measurement of residual stress in glass, using magnetophotoelasticity. Thesis, University of Nottingham, October 1979.

3 Clarke, G. P., McKenzie, H. W., and Stanley, P. The magnetophotoelastic analysis of residual stresses in thermally toughened glass. Proc. R. Soc. Lond. A. 1999, 455, 1149-1173.

4 Aben, H. K. Integrated Photoelasticity, 1979, (McGraw-Hill, New York).

5 Gibson, S., Jewell, G. W., and Tomlinson, R. A. Full-field pulsed magnetopolariscope - experimental implementation. J. Strain Analysis, 2006, 41(2), 171-182.

6 Gibson, S., and Tomlinson, R. A. Experimental determination of through-thickness residual stresses in toughened glass. In Proceedings of the BSSM International Conference on Advances in Experimental Mechanics, Stratford-upon-Avon, 27-29 August 2002, pp. 77-80. 
7 Gibson, S. Experimental determination of the through-thickness residual stress distribution in glass. Thesis, University of Sheffield, May 2004.

8 Hundhammer, I., Lenhart, A., Pontasch, D., and Weißmann, R. Stress measurement in transparent materials using scattered laser light. Glass Sci. Technol., 2002, 75, 236-242.

9 Gibson, S. Experimental determination of the through-thickness residual stress distribution in glass. Thesis, University of Sheffield, May 2004.

10 Riley, C. D. The design of magnetizing fixtures and powder aligning systems for bonded NdFeB permanent magnets. Thesis, University of Sheffield, 1996.

11 Grossinger, R., Jewell, G. W., Dudding, J., and Howe, D. Pulsed field magnetometry. IEEE Trans. Magnetics, 1993, MAG 29(6), 2980-2982.

12 Asano, T., Sakai, Y., Kido, G., Inoue, K., and Maeda, H. Development of wire wound pulsed magnet. Physica B: Condensed Matter, 1995, $211(1-4), 46-49$.

13 Azzam, R. M. A. A simple Fourier photopolarimeter with rotating polariser and analyser for measuring
Jones and Mueller matrices. Opt. Communications, 1978, 25, 137-140.

14 Berezhna, S., Berezhnyy, I., and Takashi, M. High resolution birefringence imaging in threedimensional stressed models by Fourier polarimetry. Appl. Optics, 2001, 40(28), 4940-4946.

15 Tomlinson, R. A. and Patterson, E. A. Measurement of Poincaré characteristic parameters using phase stepping. Expl Mechanics, 2002, 42(1), 43-50.

16 Theocaris, P. S. and Gdoutos, E. E. Matrix Theory of Photoelasticity, 1979 (Springer-Verlag, Berlin).

\section{APPENDIX}

\section{Notation}

$B \quad$ magnetic flux density (T)

F $\quad$ Faraday rotation (deg)

$t$ thickness of the medium (mm)

$V \quad$ Verdet constant (deg/T mm) 